\title{
Microscopic investigation of the weakly correlated noncentrosymmetric superconductor $\mathrm{SrAuSi}_{3}$
}

\author{
N. Barbero, ${ }^{1}$ P. K. Biswas, ${ }^{2, *}$ M. Isobe, ${ }^{3}$ A. Amato, ${ }^{4}$ E. Morenzoni, ${ }^{4}$ A. D. Hillier, ${ }^{2}$ H.-R. Ott, ${ }^{1,4}$ J. Mesot, ${ }^{1,4}$ and T. Shiroka ${ }^{1,4}$ \\ ${ }^{1}$ Laboratorium für Festkörperphysik, ETH Zürich, CH-8093 Zurich, Switzerland \\ ${ }^{2}$ ISIS Pulsed Neutron and Muon Source, STFC Rutherford Appleton Laboratory, Harwell Campus, \\ Didcot, Oxfordshire OX11 0QX, United Kingdom \\ ${ }^{3}$ Superconducting Properties Unit, National Institute for Materials Science, 1-1 Namiki, Tsukuba, Ibaraki 305-0044, Japan \\ ${ }^{4}$ Paul Scherrer Institut, $\mathrm{CH}-5232$ Villigen PSI, Switzerland
}

(Received 13 November 2017; published 3 January 2018)

\begin{abstract}
$\mathrm{SrAuSi}_{3}$ is a noncentrosymmetric superconductor (NCS) with $T_{c}=1.54 \mathrm{~K}$, which to date has been studied only via macroscopic techniques. By combining nuclear-magnetic-resonance and muon-spin-rotation measurements, we investigate both the normal and the superconducting phase of $\mathrm{SrAuSi}_{3}$ at a local level. In the normal phase, our data indicate a standard metallic behavior with weak electron correlations and a Korringa constant $S_{\exp }=1.31 \times$ $10^{-5} \mathrm{sK}$. The latter, twice the theoretical value, can be justified by the Moriya theory of exchange enhancement. In the superconducting phase, the material exhibits conventional BCS-type superconductivity with a weak-coupling $s$-wave pairing, a gap value $\Delta(0)=0.213(2) \mathrm{meV}$, and a magnetic penetration depth $\lambda(0)=398(2) \mathrm{nm}$. The experimental proof of weak correlations in $\mathrm{SrAuSi}_{3}$ implies that correlation effects can be decoupled from those of antisymmetric spin-orbit coupling, thus enabling accurate band-structure calculations in the weakly correlated NCSs.
\end{abstract}

DOI: 10.1103/PhysRevB.97.024501

\section{INTRODUCTION}

The absence of inversion symmetry in the crystal lattice of certain materials introduces antisymmetric spin-orbit coupling (ASOC) in the conduction-electron ensemble. In the case of superconductivity, this may result in mixed-parity phases [1], which have increasingly attracted the attention of the scientific community, especially after the discovery of the first strongly correlated noncentrosymmetric superconductor (NCS) $\mathrm{CePt}_{3} \mathrm{Si}$ [2]. With $T_{\mathrm{N}}=2.2 \mathrm{~K}$ and $T_{c}=0.75 \mathrm{~K}$, it was argued that in $\mathrm{CePt}_{3} \mathrm{Si}$ the magnetic fluctuations induce a nonstandard pairing mechanism.

Since $\mathrm{CePt}_{3} \mathrm{Si}$, several new compounds belonging to different NCS classes have been discovered [1]. In particular, analogous ternary compounds with weak or negligible electronic correlations have been found to exhibit conventional or mixed-pairing superconductivity (SC) and are actively under investigation [3]. The study of NCSs with only weak electronic correlations is key to understanding the superconductivity in noncentrosymmetric compounds. Indeed, weak correlations make it possible to disentangle the ASOC, which reflects the lack of inversion symmetry, from the role of correlations. Accurate band-structure calculations, focused exclusively on the ASOC splitting of electronic bands, are therefore within reach.

$\mathrm{SrAuSi}_{3}$, recently synthesized in the form of polycrystals by means of high-pressure techniques [4], crystallizes in the $\mathrm{BaNiSn}_{3}$-type $\mathrm{NCS}$ structure (tetragonal with space group $I 4 \mathrm{~mm}$, SG no. 107). The first magnetization and transport measurements determined the onset of superconductivity at $T_{c}=1.54 \mathrm{~K}$ and an upper critical field $\mu_{0} H_{c 2}(0)$ of $0.18 \mathrm{~T}$.

\footnotetext{
*pabitra.biswas@stfc.ac.uk
}

Subsequently, two theoretical first-principles calculations on $\mathrm{SrAuSi}_{3}$ revealed lattice constants and an electronic structure that are only in partial agreement with the experimental data $[5,6]$. Further measurements of the specific heat and band calculations [7] suggested a nodeless gap, supporting the hypothesis that $\mathrm{SrAuSi}_{3}$ is an $s$-wave BCS-type superconductor. Additional theoretical calculations [8] claimed an electronphonon coupling parameter $\lambda=0.47$ and attributed the pairing mainly to the $p$ electrons of $\mathrm{Si}$. $\mathrm{SrAuSi}_{3}$ may be compared with $\mathrm{BaPtSi}_{3}$, an isostructural compound with very similar lattice parameters, Debye temperature, and electron-phonon coupling. Reference [9] reports that $\mathrm{BaPtSi}_{3}$ is a standard weakcoupling conventional superconductor with $2 \Delta / k_{\mathrm{B}} T_{c}=3.5$.

Below, we report a microscopic study of $\mathrm{SrAuSi}_{3}$ by employing nuclear-magnetic-resonance (NMR) and muon-spinrotation $(\mu \mathrm{SR})$ measurements. We probed both its normal and superconducting phase, and show that $\mathrm{SrAuSi}_{3}$ is a weakly correlated metal turning into a conventional superconductor.

Section II offers an overview of the employed experimental techniques, including the relevant settings and conditions. In Sec. III A the electronic correlations in the normal phase are studied via NMR experiments, whereas in Sec. IIIB the SC phase is investigated by $\mu \mathrm{SR}$, and the respective main $\mathrm{SC}$ parameters are evaluated quantitatively. Finally, Sec. IV offers conclusions and a general discussion regarding open questions about the class of weakly correlated NCSs, with particular reference to $\mathrm{SrAuSi}_{3}$.

\section{EXPERIMENTAL DETAILS}

Samples of $\mathrm{SrAuSi}_{3}$ were prepared using the solid-state reaction method under $6 \mathrm{GPa}$ at $1000-1200{ }^{\circ} \mathrm{C}[4,7]$. Preliminary $\mathrm{X}$-ray diffraction (XRD) measurements were used to verify 


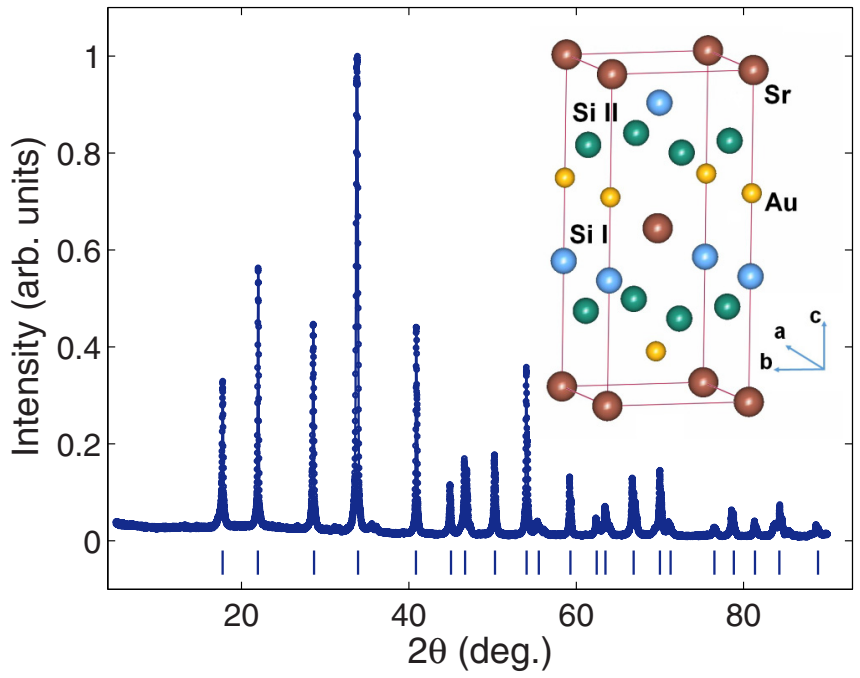

FIG. 1. XRD pattern of the polycrystalline sample. Data were acquired using the $K \alpha$ line of $\mathrm{Cu}(40 \mathrm{kV}, 15 \mathrm{~mA})$ and fitted by following a standard Rietveld-refinement procedure.

the sample's crystal structure (see Fig. 1). No spurious phases above a $0.1 \%$ limit could be detected, and the already known tetragonal $I 4 \mathrm{~mm}$ structure was confirmed [4].

The NMR investigations included line-shape and spinlattice relaxation $\left(T_{1}\right)$ measurements in an applied magnetic field of $7.0571 \mathrm{~T}$. The most suitable nucleus for our study was ${ }^{29} \mathrm{Si}$ (spin $I=1 / 2$, natural abundance $4.7 \%$, and a Larmor frequency of $59.685 \mathrm{MHz}$ ). The ${ }^{29} \mathrm{Si}$ NMR spectra were obtained via the fast Fourier transformation (FFT) of the spinecho signals generated by $\pi / 2-\pi$ rf pulses of $11-$ and $22-\mu \mathrm{s}$ duration, respectively, with a $50-\mu$ s spacing. The recycle delays ranged from $0.2 \mathrm{~s}$ at room temperature up to $10 \mathrm{~s}$ at $2 \mathrm{~K}$. To improve the quality of the spectra, frequency sweeps with 20$\mathrm{kHz}$ steps were used. The nuclear spin-lattice relaxation times $T_{1}$ were measured at both peak frequencies, corresponding to two inequivalent $\mathrm{Si}$ sites, by using the inversion-recovery method with spin-echo detection at variable delays. Due to the intermediate sensitivity of ${ }^{29} \mathrm{Si}$ nuclear spins, several scans had to be accumulated (from 200 at $2 \mathrm{~K}$ up to $1 \times 10^{4}$ at $290 \mathrm{~K})$.

The local-field distribution within the material was monitored with the very sensitive $\mu$ SR technique. The transversefield (TF) $\mu \mathrm{SR}$ technique is often used to measure the value and the temperature dependence of the magnetic-penetration depth $\lambda$ in the vortex state of type-II superconductors [10]. $\lambda^{-2}(T)$ is proportional to the density of superconducting carriers $n_{\mathrm{s}}$, hence it provides information on the character of the gap in the electronic excitation spectrum below $T_{c}$. The $\mathrm{TF}-\mu \mathrm{SR}$ experiments were carried out at the low-temperature (LTF) instrument of the $\pi \mathrm{M} 3$ beam line at the Paul Scherrer Institute (Villigen, Switzerland). To avoid flux-pinning effects, the sample was cooled to the base temperature in an applied magnetic field of $20 \mathrm{mT}$. The data were collected between 0.02 and $1.8 \mathrm{~K}$ while warming the sample in a steady field. Typically $\sim 12 \times 10^{6}$ muon-decay events were collected for each spectrum. The TF- $\mu$ SR data were analyzed by means of the software package MUSRFIT [11].

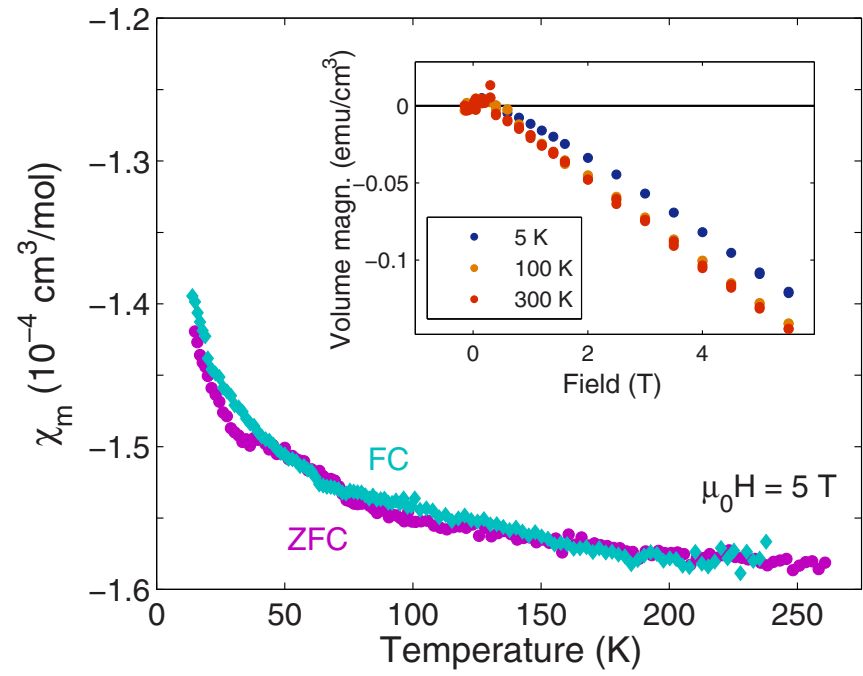

FIG. 2. Magnetometry $\chi_{m}(T)$ data measured at $\mu_{0} H=5 \mathrm{~T}$ in the normal phase (down to $4 \mathrm{~K}$ ). The inset shows the magnetization $M(H)$ curves at three selected temperatures (5, 100, and $300 \mathrm{~K})$.

Given the low $T_{c}=1.54 \mathrm{~K}$ and the modest upper critical field of $0.18 \mathrm{~T}$, an NMR study of $\mathrm{SrAuSi}_{3}$ in the superconducting phase is quite challenging. The required spin-polarizing field suppresses superconductivity in fields exceeding $H_{c 2}(0)$. If the chosen applied field were substantially weaker, the ${ }^{29} \mathrm{Si}$ Larmor frequency would have been very low, resulting in an extremely poor $\mathrm{S} / \mathrm{N}$ ratio. On the other hand, due to the intrinsic spin-polarization of muon beams, TF- $\mu$ SR can be successfully used in the SC phase even at small applied fields $(\sim 20 \mathrm{mT})$, yet it is much less sensitive in the normal phase. In view of these considerations, we chose NMR to probe the normal phase and $\mu \mathrm{SR}$ for the SC-phase of $\mathrm{SrAuSi}_{3}$.

\section{RESULTS AND DISCUSSION}

\section{A. Normal-phase nuclear-magnetic-resonance investigations}

For a preliminary characterization of the electronic and magnetic properties of the normal phase, we measured the sample's magnetization $M(T, H)$ with a commercial MPMS (magnetic property measurement system) XL setup. With a typical $\chi_{\text {dia }}$ of $-1.6 \times 10^{-4} \mathrm{~cm}^{3} / \mathrm{mol}$ (see Fig. 2), the $\chi_{m}(T)=M(T) / H$ data of $\mathrm{SrAuSi}_{3}$ mimic those of $\mathrm{SrPt}_{3} \mathrm{P}$ [12], a centrosymmetric superconductor, and of the diamagnetic Au $\left(-1.2 \times 10^{-4} \mathrm{~cm}^{3} / \mathrm{mol}\right)$ [13]. The inset of Fig. 2 confirms that the bulk of the sample is predominantly diamagnetic. The small positive response at low fields suggests the presence of a small amount $(<0.1 \%)$ of impurities, reflected also in the low-field hysteretic cycle (not shown).

From the published structure-data file [4], Si occupies two inequivalent lattice sites: $\mathrm{Si}-\mathrm{I}$ (two atoms per unit cell, i.e., one internal and four at the edges) and Si-II (four atoms per unit cell, i.e., eight atoms on the faces). From the 2:4 multiplicity ratio, we expect two resonance lines, related to the $\mathrm{Si}$-I and $\mathrm{Si}$-II nuclei, with an ideal ratio of 2 for the peak areas. The observed spectra were fitted by assuming a simple model [see Eq. (1)], 


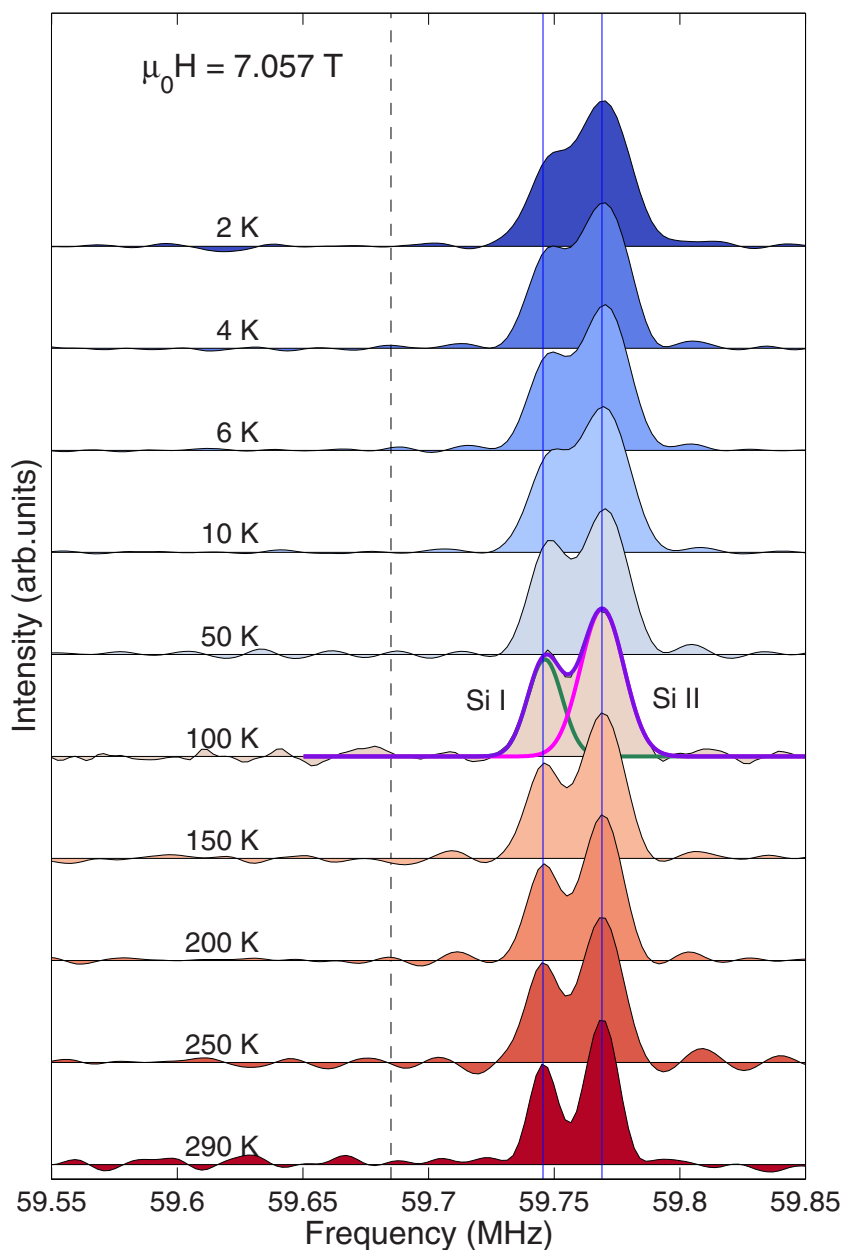

FIG. 3. ${ }^{29} \mathrm{Si}$ line shapes measured at 7.06 T. The spectra were fitted by a two-Gaussian model, as shown for $T=100 \mathrm{~K}$. The dashed black line corresponds to the reference Larmor frequency of ${ }^{29} \mathrm{Si}$, whereas the solid blue lines correspond to the frequencies of the two peaks at room temperature (RT).

consisting of the sum of two Gaussian peaks, as illustrated in Fig. 3 for $T=100 \mathrm{~K}$ :

$$
I(f)=a_{1} \exp \left[-\frac{\left(f-f_{1}\right)^{2}}{2 \sigma_{1}^{2}}\right]+a_{2} \exp \left[-\frac{\left(f-f_{2}\right)^{2}}{2 \sigma_{2}^{2}}\right],
$$

where $a_{i}(i=1,2)$ are the normalization factors, $f_{i}$ are the peak maxima, and $w_{i}$ are their full widths at half-maximum (FWHM), with $w_{i}=2(2 \ln 2)^{1 / 2} \sigma_{i} \simeq 2.35 \sigma_{i}$.

As the temperature is lowered from 300 to $5 \mathrm{~K}$, the shift of the Si-I NMR line varies from $0.1 \%$ to $0.105 \%$, whereas that of the Si-II lines varies from $0.14 \%$ to $0.142 \%$ (see Fig. 4). These shift values are similar to those reported in the literature for typical metals such as $\mathrm{Na}$ [14]. Indeed, a nonvarying value in the normal phase is commonly observed in simple metals whose electronic susceptibility $\chi_{P}$ varies only weakly with temperature [12]. In both cases, a tiny gradual enhancement of the line shift is observed below $100 \mathrm{~K}$, probably due to a small change in the electronic environment (see Fig. 4).

As shown in Fig. 5, both NMR lines broaden upon lowering the temperature, with FWHM values changing smoothly from

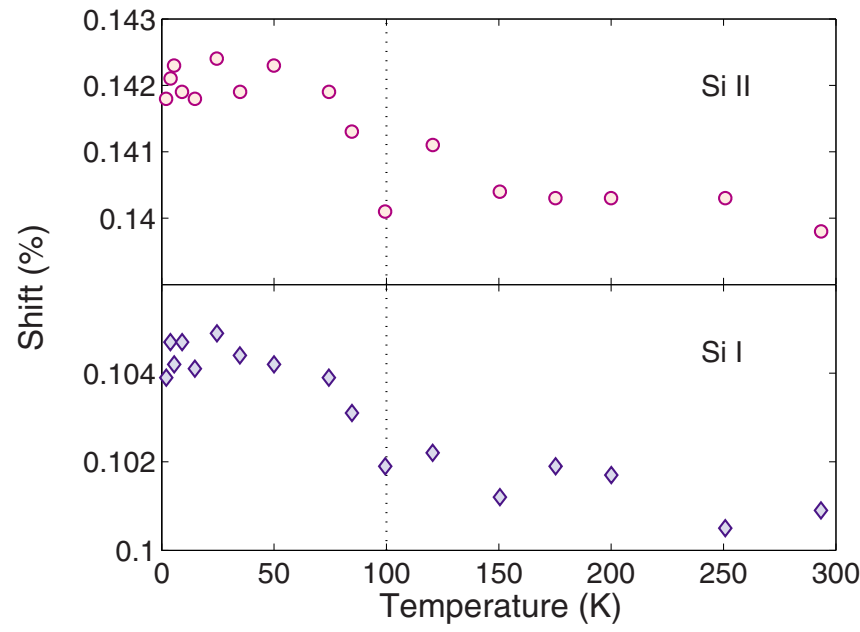

FIG. 4. Shifts of the two ${ }^{29} \mathrm{Si}$ NMR lines vs temperature.

about 6 to $8 \mathrm{kHz}$ for $\mathrm{Si}-\mathrm{I}$ and from 7 to $9 \mathrm{kHz}$ for $\mathrm{Si}-\mathrm{II}$ sites. From the fits we estimate a ratio of peak areas of $1.8 \pm 0.2$, to be compared with the expected value of 2 , as explained above. The small discrepancy is most likely due to a $T_{1}$-induced signal-intensity modulation, reflecting the different relaxation rates of nuclei at the $\mathrm{Si}-\mathrm{I}$ and $\mathrm{Si}$-II sites, whose $T_{1}$ values differ by a factor of 2 at room temperature.

In the normal phase, we find a linear variation of $1 / T_{1}(T)$, i.e., a Korringa behavior typical of simple metals (see Fig. 6). The average $T_{1} T$ value is $6.49 \mathrm{sK}$, which, by considering the measured $0.14 \%$ Knight shift, implies an experimental Korringa constant $S_{\exp } \equiv T_{1} T K^{2}=1.31 \times 10^{-5} \mathrm{sK}$, twice the theoretical value $S_{0}=\hbar\left(\gamma_{e} / \gamma_{\mathrm{Si}}\right)^{2} /\left(4 \pi k_{\mathrm{B}}\right)=6.66 \times 10^{-6} \mathrm{sK}$. From the relative Korringa ratio $\mathcal{K} \equiv S_{0} / S_{\text {exp }}=0.508$, one can evaluate the enhancement of the static and dynamic spin susceptibilities, driven by the $q$-independent electron-electron interaction potential [15] $\zeta=\hbar^{2} \gamma_{e}^{2} \alpha / 2 \pi \chi_{s 0}$, where $\gamma_{e}$ is the gyromagnetic ratio of the electron, $\chi_{s 0}$ is the spin susceptibility,

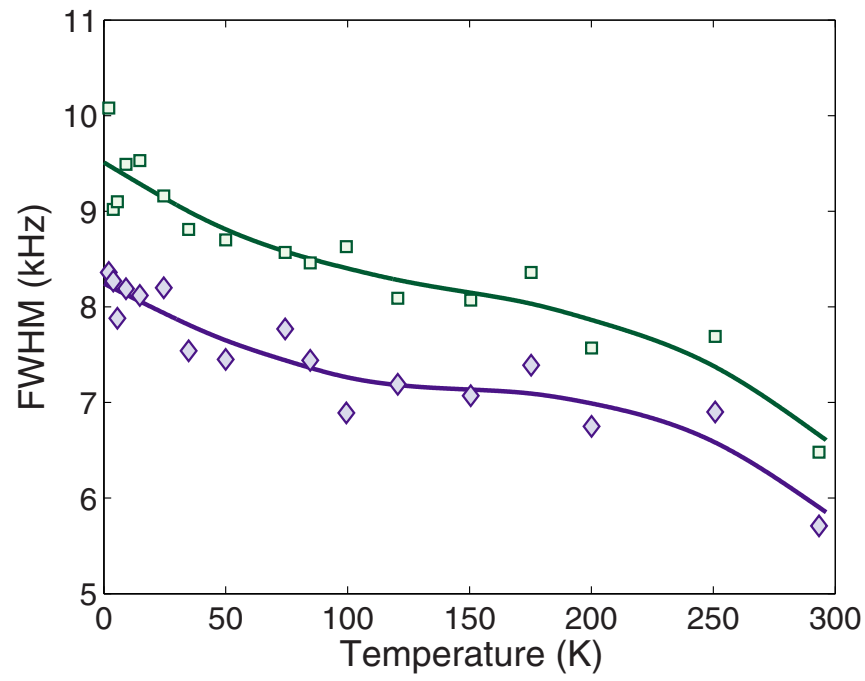

FIG. 5. FWHM vs $T$ plots of both ${ }^{29} \mathrm{Si}$ NMR lines indicate a gradual broadening as the temperature is lowered. 


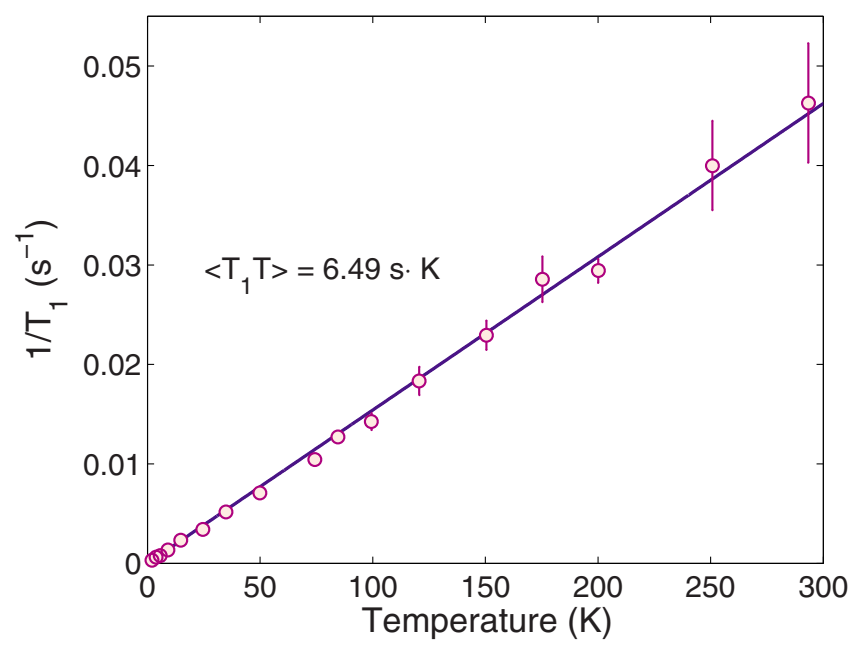

FIG. 6. $1 / T_{1}$ vs $T$ for the ${ }^{29} \mathrm{Si}$ NMR of the Si-II site.

and $\alpha$ is a parameter that is uniquely defined by the value of $\mathcal{K}$ via $[14,16]$

$$
\mathcal{K}(\alpha)=2(1-\alpha)^{2} \int_{0}^{1} x /[1-\alpha G(x)]^{2} d x .
$$

The quantity $G(x)$ in Eq. (2) is the Lindhard function for free electrons, i.e., $G(x)=\frac{1}{2}\left\{1+\left[\left(1-x^{2}\right) / 2 x\right] \ln (|1+x| / \mid 1-\right.$ $x \mid)\}$, with the normalized momentum $x=q /\left(2 k_{\mathrm{F}}\right)$, where $k_{\mathrm{F}}$ is the Fermi momentum under the assumption of a spherical Fermi surface. An analytical evaluation of the $\zeta$ parameter is not possible, since the value of the spin susceptibility $\chi_{s 0}$, i.e., the Pauli susceptibility, generally cannot be estimated from the measured magnetic susceptibility $\chi_{m}=M(T, H) / H$, which contains also additional contributions due to, e.g., the core electrons and the electronic correlations. Nevertheless, the experimentally determined $\mathcal{K}(\alpha)$ suggests the presence of ferromagnetic correlations in $\mathrm{SrAuSi}_{3}$, which, however, are incompatible with the rather weak Knight shift. From the experimental $\mathcal{K}(\alpha)$ value, one can solve Eq. (2) via numerical integration and obtain $\alpha=0.34(3)$. Such an $\alpha$ value indicates weak but not negligible electron-electron interactions, in line with the Moriya theory of exchange enhancement [17]. Indeed, similar $\alpha$ and $\mathcal{K}(\alpha)$ values are found in many alkali metals, as well as in $\mathrm{Cu}$ and $\mathrm{Ag}$, all well-known realizations of the nearly-free-electron model $[14,16]$.

\section{B. Muon-spin rotation in the superconducting phase}

TF- $\mu$ SR measurements were carried out to investigate the superconducting properties of $\mathrm{SrAuSi}_{3}$. Figures 7(a) and 7(b) show the TF- $\mu \mathrm{SR}$ time spectra, collected at 1.6 and $0.02 \mathrm{~K}$ in a magnetic field of $20 \mathrm{mT}$. While the $\mu \mathrm{SR}$ time spectrum obtained at $1.6 \mathrm{~K}$ in the normal state shows almost no relaxation, data collected at $0.02 \mathrm{~K}$ in the superconducting state reveal pronounced damping in the $\mu \mathrm{SR}$ time spectra due to the inhomogeneous field distribution generated by the formation of a vortex lattice. The TF- $\mu$ SR time spectra were analyzed using the oscillatory Gaussian decay function [10]:

$$
\begin{aligned}
A^{\mathrm{TF}}(t)= & A(0) \exp \left(-\sigma^{2} t^{2} / 2\right) \cos \left(\gamma_{\mu} B_{\mathrm{int}} t+\phi\right) \\
& +A_{\mathrm{bg}}(0) \cos \left(\gamma_{\mu} B_{\mathrm{bg}} t+\phi\right),
\end{aligned}
$$

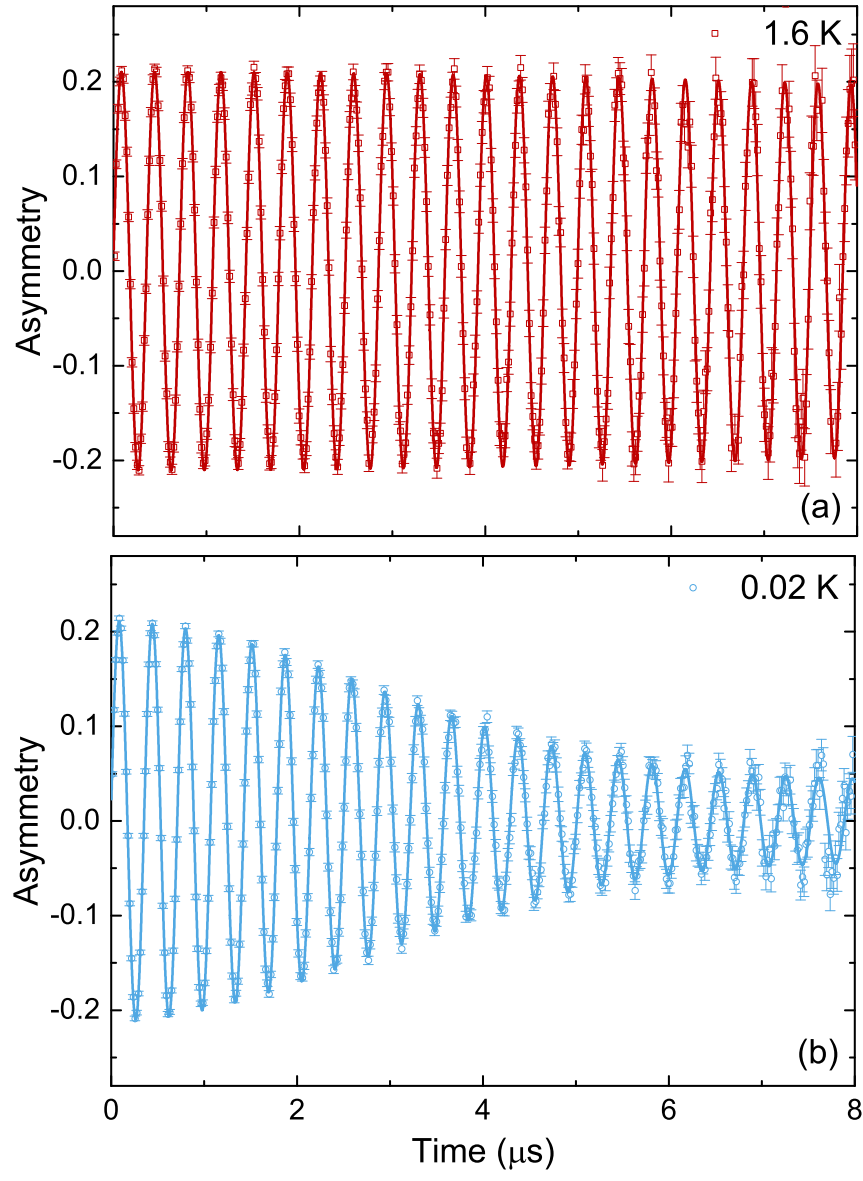

FIG. 7. Parts (a) and (b) show the TF- $\mu \mathrm{SR}$ time spectra of $\mathrm{SrAuSi}_{3}$, collected at 1.6 and $0.02 \mathrm{~K}$ (above and below $T_{\mathrm{c}}$ ) in an applied field of $20 \mathrm{mT}$. The solid lines are least-squares fits to the asymmetry spectra according to Eq. (3).

where the fit parameters $A(0)$ and $A_{\mathrm{bg}}(0)$ are the initial asymmetries of the sample and background signals, $\gamma_{\mu} / 2 \pi=$ $13.55 \mathrm{kHz} / \mathrm{G}$ is the muon gyromagnetic ratio [10], $B_{\text {int }}$ and $B_{\mathrm{bg}}$ are the internal and background magnetic fields, $\phi$ is the initial phase of the muon precession signal, and $\sigma$ is the Gaussian muon-spin-relaxation rate. The last term in Eq. (3) represents the background signal that originates mostly from muons hitting the silver sample holder and is considered as nonrelaxing within the muon time window [10]. The signalto-background ratio is $A_{\mathrm{bg}}(0) / A(0)=0.21$.

Figure 8(a) shows the temperature dependence of $\sigma$ of $\mathrm{SrAuSi}_{3}$ for an applied field of $20 \mathrm{mT}$, exhibiting a pronounced increase just below $T=T_{\mathrm{c}}$. Figure 8(b) shows the temperature dependence of the internal magnetic field at the muon site, revealing the expected diamagnetic shift and confirming the bulk-type superconductivity in this material. The superconducting contribution $\sigma_{\mathrm{sc}}$ can be obtained by quadratically subtracting the nuclear moment contribution $\sigma_{\mathrm{nm}}$ (measured above $T_{\mathrm{c}}$ ) from the total $\sigma$, as $\sigma_{\mathrm{sc}}{ }^{2}=\sigma^{2}-\sigma_{\mathrm{nm}}{ }^{2}$. Since we do not expect any structural transition in $\mathrm{SrAuSi}_{3}$ over the temperature range of the $\mathrm{TF}-\mu \mathrm{SR}$ study, $\sigma_{\mathrm{nm}}$ is assumed to be temperature-independent. In an isotropic type-II superconductor with a hexagonal Abrikosov vortex lattice, the magnetic penetration depth $\lambda$ is related to $\sigma_{\mathrm{sc}}$ by the Brandt 


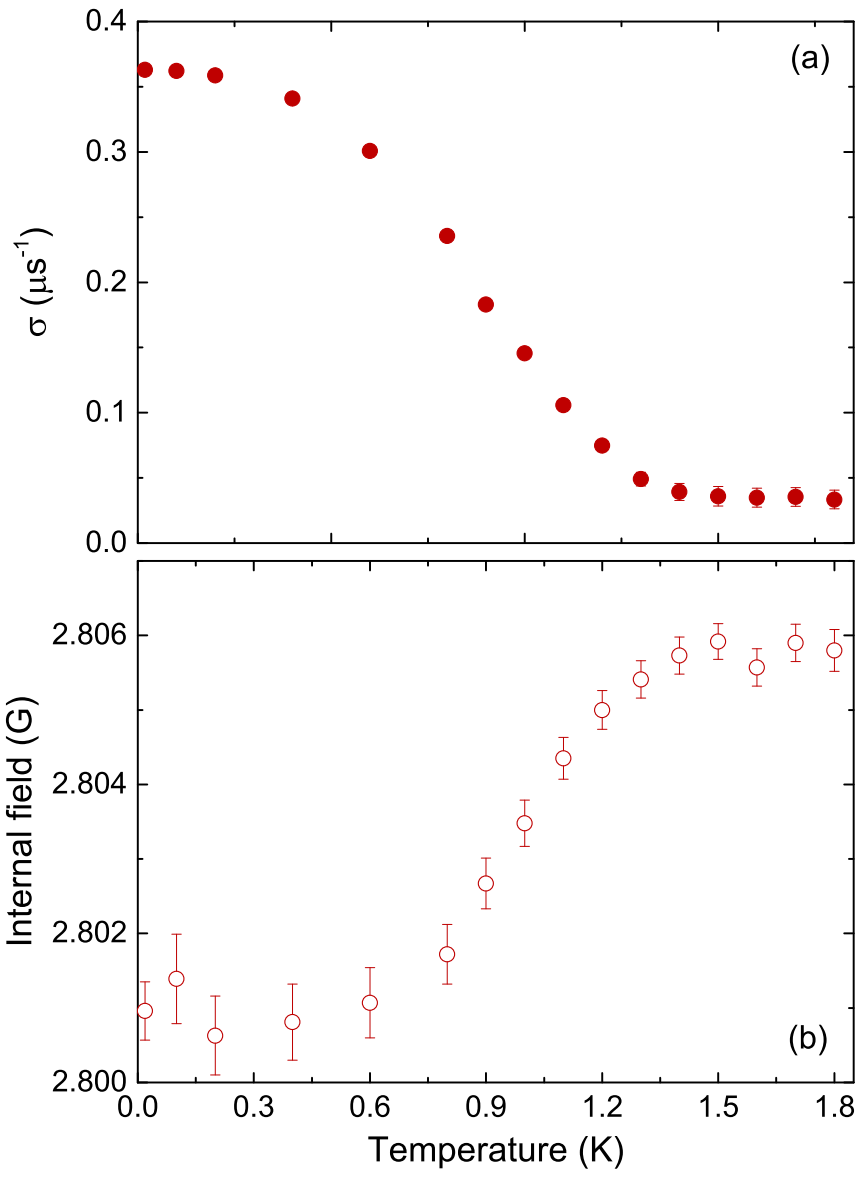

FIG. 8. (a) Temperature dependence of the muon depolarization rate $\sigma$ of $\mathrm{SrAuSi}_{3}$ in an applied magnetic field of $20 \mathrm{mT}$. (b) Diamagnetic shift of the internal field experienced by the muons just below $T_{\mathrm{c}}$.

equation [18]:

$$
\begin{aligned}
\sigma_{\mathrm{sc}}\left(\mu \mathrm{s}^{-1}\right)(T)= & 4.854 \times 10^{4}\left(1-\frac{H}{H_{\mathrm{c} 2}(T)}\right) \\
& \times\left[1+1.21\left(1-\sqrt{\frac{H}{H_{\mathrm{c} 2}(T)}}\right)^{3}\right] \\
& \times \lambda^{-2}\left(\mathrm{~nm}^{-2}\right),
\end{aligned}
$$

where $H$ and $H_{\mathrm{c} 2}(T)$ are the applied and upper critical fields, respectively.

Figure 9 shows $\lambda^{-2}(T)$ of $\mathrm{SrAuSi}_{3}$, calculated using Eq. (4). The temperature dependence of $H_{\mathrm{c} 2}(T)$ was taken from Ref. [4]. $\lambda^{-2}$ is proportional to the effective superfluid density, $\rho_{\mathrm{s}}$. Therefore, $\lambda^{-2}(T)$ provides information about the magnitude of the superconducting gap and the symmetry of the gap structure. Figure 9 reveals that $\rho_{\mathrm{s}}$ of $\mathrm{SrAuSi}_{3}$ is nearly constant below $T_{\mathrm{c}} / 3 \approx 0.5 \mathrm{~K}$, suggesting a nodeless superconducting gap in this material. A good fit to $\lambda^{-2}(T)$ can be achieved with a single-gap BCS $s$-wave model $[19,20]$ (solid line in Fig. 9) using the functional form

$$
\frac{\lambda^{-2}(T)}{\lambda^{-2}(0)}=1+2 \int_{\Delta(T)}^{\infty}\left(\frac{\partial f}{\partial E}\right) \frac{E \mathrm{~d} E}{\sqrt{E^{2}-\Delta(T)^{2}}} .
$$

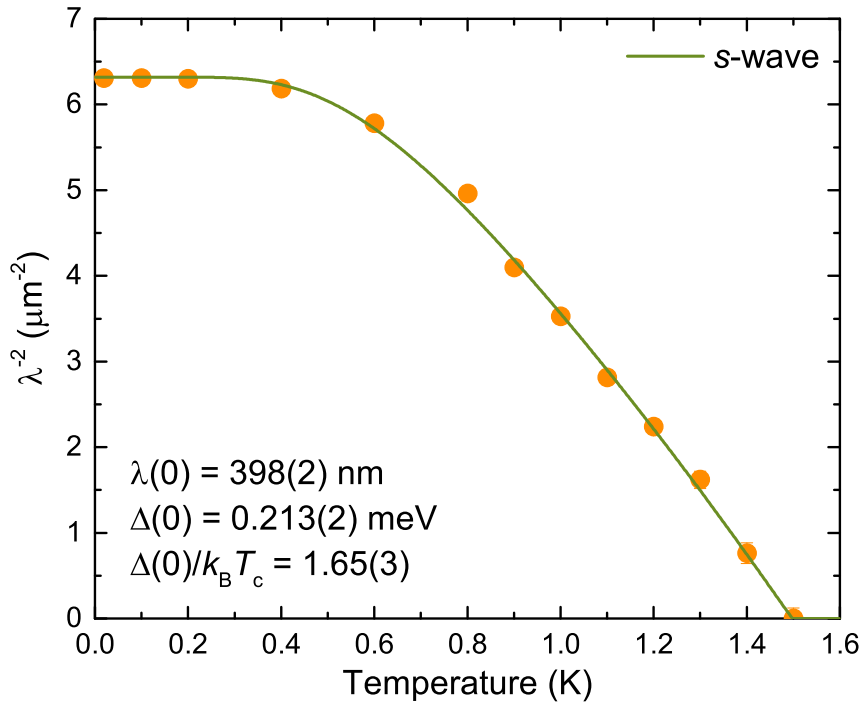

FIG. 9. The temperature dependence of $\lambda^{-2}(T)$. The solid line is a fit to the $\lambda^{-2}(T)$ with the weak-coupling BCS $s$-wave model.

Here $\lambda^{-2}(0)$ is the zero-temperature value of the magnetic penetration depth and $f=\left[1+\exp \left(E / k_{\mathrm{B}} T\right)\right]^{-1}$ denotes the Fermi function. The BCS temperature dependence of the superconducting gap function can be approximated by [21]

$$
\Delta(T)=\Delta(0) \tanh \left\{1.82\left[1.018\left(\frac{T_{c}}{T}-1\right)\right]^{0.51}\right\},
$$

where $\Delta(0)$ is the gap magnitude at zero temperature. The fit yields $T_{c}=1.49(1) \mathrm{K}, \lambda(0)=398(2) \mathrm{nm}$, and $\Delta(0)=$ $0.213(2) \mathrm{meV}$. The ratio $\Delta(0) / k_{\mathrm{B}} T_{\mathrm{c}}=1.65(3)$, which is lower than the BCS value of 1.76 , confirms that $\mathrm{SrAuSi}_{3}$ is a weakcoupling superconductor.

\section{CONCLUSIONS}

A combined study of the normal and superconducting phase of $\mathrm{SrAuSi}_{3}$ was performed through NMR and $\mu \mathrm{SR}$ in TF mode measurements, respectively. In the normal phase, this compound exhibits a Korringa behavior with enhanced electron-exchange behavior, in line with the Moriya theory mentioned above. The temperature dependence of the London penetration depth $\lambda$ was evaluated from the TF- $\mu$ SR time spectra. $\lambda(T)$ can be modeled well by assuming a single-gap BCS $s$-wave scenario with $\Delta(0)=0.213(2) \mathrm{meV}$, which suggests that $\mathrm{SrAuSi}_{3}$ is a nodeless superconductor. The gap-to- $T_{c}$ ratio provides evidence for weak-coupling superconductivity in $\mathrm{SrAuSi}_{3}$. The magnetic penetration depth was estimated as $\lambda(0)=398(2) \mathrm{nm}$.

Single-band fully gapped BCS superconductivity and weak electronic correlation confirm the small splitting of the bands in the vicinity of the Fermi level $E_{\mathrm{F}}$, visible only in proximity of the Brillouin-zone edges [8]. This hints at a small ASOC that, along with weak correlations, may hinder the singlet-triplet mixing. Although a mixed-parity pairing is predicted to be a general feature of NCSs with ASOC, many weakly correlated compounds exhibit a rather simple behavior [3], as reported 
here for the $\mathrm{SrAuSi}_{3}$ case. This general trend can be explained by considering that the triplet component is very weak, therefore two gaps of very similar magnitude will be dominated by spin-singlet pairing, i.e., a mechanism indistinguishable from ordinary $s$-wave superconductors. When looking for new systems exhibiting singlet-triplet mixing, a simple comparison of the relative energies of ASOC and SC condensate is often not sufficient, since also pairing interactions in both the singlet and triplet channels are required for the occurrence of parity mixing. This is evidenced by the fact that weakly correlated NCSs do not exhibit mixing, whereas their strongly correlated counterparts (including Ce- and other heavy-electron based systems) may do so [3].

In perspective, further experimental and theoretical work is required in order to confirm the occurrence of mixed singlet- triplet pairing, since the role of ASOC and that of the strength of electronic correlations still remains poorly understood. Furthermore, due to their nontrivial band structure, topological SC states are expected in several NCSs. Investigations by local probes, such as those reported here, remain crucial to support or rule out new hypotheses aiming to explain the intriguing properties of noncentrosymmetric superconductors.

\section{ACKNOWLEDGMENTS}

This work was financially supported in part by the Schweizerische Nationalfonds zur Förderung der Wissenschaftlichen Forschung (SNF) and by the Japan Society for the Promotion of Science (JSPS) through Grants-in-Aid for Scientific Research (Grant No. 16K06712).
[1] E. Bauer and M. Sigrist, Non-Centrosymmetric Superconductors (Springer Verlag, Berlin, 2012).

[2] E. Bauer, G. Hilscher, H. Michor, Ch. Paul, E. W. Scheidt, A. Gribanov, Yu. Seropegin, H. Noël, M. Sigrist, and P. Rogl, Heavy Fermion Superconductivity and Magnetic Order in Noncentrosymmetric $\mathrm{CePt}_{3} \mathrm{Si}$, Phys. Rev. Lett. 92, 027003 (2004).

[3] M. Smidman, M. B. Salamon, H. Q. Yuan, and D. F. Agterberg, Superconductivity and spin-orbit coupling in noncentrosymmetric materials: A review, Rep. Prog. Phys. 80, 036501 (2017).

[4] M. Isobe, H. Yoshida, K. Kimoto, M. Arai, and E. TakayamaMuromachi, $\mathrm{SrAuSi}_{3}$ : A noncentrosymmetric superconductor, Chem. Mater. 26, 2155 (2014).

[5] C. Shu, J. Long, S. Li, and W. Yang, Theoretical investigation of the newly noncentrosymmetric superconductor $\mathrm{SrAuSi}_{3}$ via first principles, J. Supercond. Nov. Magn. 28, 3235 (2015).

[6] C. Shu, J. Long, S. Li, and W. Yang, Theoretical investigation of the mechanical and thermodynamics properties of the noncentrosymmetric superconductor $\mathrm{SrAuSi}_{3}$, J. Alloys Compd. 650, 553 (2015).

[7] M. Isobe, M. Arai, and N. Shirakawa, Superconductivity in noncentrosymmetric SrAuSi 3 , Phys. Rev. B 93, 054519 (2016).

[8] E. Arslan, E. Karaca, H. M. Tütüncü, A. Başoglu, and G. P. Srivastava, Theoretical Investigation of superconductivity in $\mathrm{SrAuSi}_{3}$ and $\mathrm{SrAu}_{2} \mathrm{Si}_{2}$, J. Phys. Chem. Solids 95, 65 (2016).

[9] E. Bauer, R. T. Khan, H. Michor, E. Royanian, A. Grytsiv, N. Melnychenko-Koblyuk, P. Rogl, D. Reith, R. Podloucky, E.-W. Scheidt, W. Wolf, and M. Marsman, Baptsi $i_{3}$ : A noncentrosymmetric BCS-like superconductor, Phys. Rev. B 80, 064504 (2009).
[10] J. E. Sonier, J. H. Brewer, and R. F. Kiefl, $\mu$ SR studies of the vortex state in type-II superconductors, Rev. Mod. Phys. 72, 769 (2000).

[11] A. Suter and B. M. Wojek, Musrfit: A free platform-independent framework for $\mu$ SR data analysis, Phys. Proc. 30, 69 (2012).

[12] T. Shiroka, M. Pikulski, N. D. Zhigadlo, B. Batlogg, J. Mesot, and H.-R. Ott, Pairing of weakly correlated electrons in the platinum-based centrosymmetric superconductor $\mathrm{SrPt}_{3} \mathrm{P}$, Phys. Rev. B 91, 245143 (2015).

[13] L. B. Mendelsohn, F. Biggs, and J. B. Mann, Hartree-Fock diamagnetic susceptibilities, Phys. Rev. A 2, 1130 (1970).

[14] A. Narath and H. T. Weaver, Effects of electron-electron interactions on nuclear spin-lattice relaxation rates and Knight shifts in alkali and noble metals, Phys. Rev. 175, 373 (1968).

[15] D. Pines, Paramagnetic susceptibility of conduction electrons, Phys. Rev. 95, 1090 (1954).

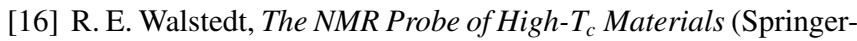
Verlag, Berlin, 2008), p. 37.

[17] T. Moriya, The effect of electron-electron interaction on the nuclear spin relaxation in metals, J. Phys. Soc. Jpn. 18, 516 (1963).

[18] E. H. Brandt, Properties of the ideal Ginzburg-Landau vortex lattice, Phys. Rev. B 68, 054506 (2003).

[19] M. Tinkham, Introduction to Superconductivity (McGraw-Hill, New York, 1975).

[20] R. Prozorov and R. W. Giannetta, Magnetic penetration depth in unconventional superconductors, Supercond. Sci. Technol. 19, R41 (2006).

[21] A. Carrington and F. Manzano, Magnetic penetration depth of $\mathrm{MgB}_{2}$, Phys. C 385, 205 (2003). 